\title{
Electrical characteristics of single- component ambipolar organic field-effect transistors and effects of air exposure on them
}

\section{$\operatorname{AUTHOR}(\mathrm{S}):$}

Sakanoue, Tomo; Yahiro, Masayuki; Adachi, Chihaya; Takimiya, Kazuo; Toshimitsu, Akio

\section{CITATION:}

Sakanoue, Tomo .... [et al]. Electrical characteristics of single-component ambipolar organic field-effect transistors and effects of air exposure on them. JOURNAL OF APPLIED PHYSICS 2008, 103(9): 094509.

\section{ISSUE DATE:}

2008-05-01

URL:

http://hdl.handle.net/2433/84566

\section{RIGHT:}

Copyright 2008 American Institute of Physics. This article may be downloaded for personal use only. Any other use requires prior permission of the author and the American Institute of Physics. 


\title{
Electrical characteristics of single-component ambipolar organic field-effect transistors and effects of air exposure on them
}

\author{
Tomo Sakanoue, ${ }^{1}$ Masayuki Yahiro, ${ }^{1}$ Chihaya Adachi, ${ }^{1, a)}$ Kazuo Takimiya, ${ }^{2}$ and \\ Akio Toshimitsu ${ }^{3}$ \\ ${ }^{1}$ Center for Future Chemistry, Kyushu University, 744 Motooka, Nishi, Fukuoka 819-0395, Japan \\ ${ }^{2}$ Department of Applied Chemistry, Graduate School of Engineering, Hiroshima University, \\ Higashi-Hiroshima 739-8527, Japan \\ ${ }^{3}$ International Innovation Center, Kyoto University, Yoshida-Honmachi, Sakyo, Kyoto 606-8501, Japan
}

(Received 5 February 2008; accepted 6 March 2008; published online 7 May 2008)

\begin{abstract}
We investigated the electrical characteristics of single-component ambipolar organic field-effect transistors (OFETs) by controlling the device structure and preparation and the measurement conditions. Six organic semiconductor materials (copper-phthalocyanine, tris-(8-hydroxyquinoline) aluminum $\left(\mathrm{Alq}_{3}\right)$, alpha-sexithiophene, 4-4'-bis-styrylphenyl, 2,7-diphenyl[1]benzothieno[3,2-b] benzothiophene, or a photopolymerized polydiacethylene derivative (PDA) were used as the active layer, and all were found to transport both holes and electrons. The PDA-based FETs had the highest hole and electron mobilities ( 0.12 and $0.025 \mathrm{~cm}^{2} / \mathrm{V} \mathrm{s}$, respectively). We also investigated the effect of air exposure on the OFETs. The hole mobility was barely affected by the exposure while the electron mobility was significantly affected. The threshold voltage for $p$-channel operation was shifted by the exposure while that for $n$-channel operation was not, indicating that the hole density in the active layer is increased by air exposure whereas the electron density is independent of air exposure. Furthermore, we prepared an $\mathrm{Alq}_{3}$-based $p$-channel OFET and investigated the effect of air exposure on it. While its operation was stable in vacuum, air exposure degraded its characteristics. These behaviors indicate that irreversible chemical reactions occur between cationic $\mathrm{Alq}_{3}$ species and oxygen or water molecules. () 2008 American Institute of Physics.
\end{abstract}

[DOI: $10.1063 / 1.2919738]$

\section{INTRODUCTION}

Organic field-effect transistors (OFETs) have recently received much attention due to their potential use in large-area and flexible electronic devices such as flexible displays and printable electronic circuits. ${ }^{1-3}$ Ambipolar OFETs are of particular interest because they can be operated in both $n$-channel and $p$-channel modes. This unique characteristic simplifies the preparation process when constructing complementary circuits. ${ }^{4,5}$ Although most ambipolar OFETs are composed of layered or mixed films of $p$-type and $n$-type semiconducting molecules, ${ }^{4-6}$ researchers have recently reported ambipolar OFETs with active layers composed of single organic semiconducting materials and the fabrication of complementary circuits. ${ }^{7,8}$ Because a balanced recombination of their holes and electrons can be achieved, such singlecomponent ambipolar OFETs are promising efficient light emitters. $^{9-15}$ Single-component ambipolar OFETs are useful for investigating the fundamental electrical characteristics of organic semiconductor materials. ${ }^{16}$ Most organic materials have thus far been empirically classified as $p$-type, $n$-type, or bipolar-type on the basis of device behavior. Furthermore, a material's highest occupied molecular orbital (HOMO) and lowest unoccupied molecular orbital (LUMO) levels, which are closely correlated with carrier injection phenomena, can be used as a basis on which to speculate to a certain extent about the material's type. However, there is no reason to

${ }^{a)}$ Electronic mail: adachi@cstf.kyushu-u.ac.jp. think that organic semiconductors would show unipolar semiconducting behavior since, unlike inorganic semiconductors, they are not intentionally doped with electron donors and acceptors. Thus, in this study, we carefully investigated the electrical characteristics of ambipolar OFETs by changing various parameters and environmental conditions, which provided insight into the unipolar semiconducting behavior of organic semiconductors.

Pioneering works have revealed that there are several key factors in the fabrication of single-component ambipolar OFETs. In the early stages of their development, such OFETs were fabricated using narrow-energy-gap (HOMO-LUMO gap of $<2.0 \mathrm{eV}$ ) semiconductors such as quinoidal terthiophene ${ }^{17}$ and poly(indenofluorene). ${ }^{5}$ Because the HOMO and LUMO levels of these materials are close to each other, the hole and electron injection barriers are not very different from those of the contacts. The injection barriers can thus be controlled by using metal contacts with a particular work function. Yasuda et al. obtained $n$-channel behavior in pentacene-based OFETs, which usually show $p$-channel unipolar behavior, by using $\mathrm{Ca}$, which has a low work function, for the source and drain contacts to reduce the electron injection barrier. ${ }^{18}$ Their results revealed that controlling the injection barriers is important to obtaining ambipolar behavior.

In 2005, a striking report was made by Chua et al., who demonstrated that most polymer semiconductors can transport both holes and electrons in carefully prepared FET structures. ${ }^{19}$ They found that hydroxyl groups on the surface 
of $\mathrm{SiO}_{2}$ dielectric layers acted as electron traps and demonstrated $n$-channel and ambipolar operations by introducing hydroxyl-free insulating polymer layers between the semiconducting layers and $\mathrm{SiO}_{2}$ layer.

Another approach to fabricating single-component ambipolar OFETs is to use organic single crystals as the active layer. de Boer et al. ${ }^{20}$ and Takahashi et al. ${ }^{21}$ prepared ambipolar OFETs using single crystals of copper and iron phthalocyanines ${ }^{20}$ rubrene, ${ }^{21}$ and tetracene. ${ }^{15}$ The findings of these studies suggest that a low trap density in the active layer, particularly for electrons, is important for obtaining ambipolar semiconducting behavior.

Carrier transport in organic semiconductors is significantly affected by the environmental conditions. For example, it has been shown that the presence of oxygen converts the polarities of OFETs fabricated of titanylphthalocyanine or fullerene from $n$ - to $p$-channel. ${ }^{24,25} \mathrm{By}$ preparing OFETs in an ultrahigh vacuum and taking successive electrical measurements without breaking the vacuum, we can evaluate the intrinsic carrier transport properties and determine how active gases, such as oxygen and water vapor, affect OFET electrical characteristics. ${ }^{22-26}$

These earlier works have shown that most organic semiconductors are intrinsically able to transport both holes and electrons in FET structures when the key factors (semiconductor/metal and semiconductor/dielectric interfaces, organic semiconductor purity, device preparation procedures, and measurements conditions) are controlled to reduce the number of injection barriers and traps. In previous work, by controlling these factors, we demonstrated ambipolar operations of OFETs even when we used a wide-energygap blue-emitting organic semiconductor and obtained efficient blue emission. ${ }^{14}$

To investigate the electrical characteristics of ambipolar OFETs, we used six representative organic semiconductor materials as the active layer and prepared the samples without breaking the high vacuum during organic and metal deposition and electrical measurement. The metals for the source and drain contacts were changed to investigate the effects of the injection barriers on ambipolar behavior. Samples were also exposed to air to investigate how carrier transport in organic semiconductors is affected by environmental conditions.

\section{EXPERIMENT}

The molecular structures of the six organic semiconductors used as the active layer are shown in Fig. 1(a): copper-phthalocyanine (CuPc) (Aldrich Co.), tris-(8hydroxyquinoline) aluminum $\left(\mathrm{Alq}_{3}\right)$ (Nippon Steel Chemical Co.), alpha-sexithiophene ( $\alpha$-6T) (Aldrich Co.), 4-4'-bisstyrylphenyl $\quad$ (BSBP) ${ }^{14}$ 2,7-diphenyl[1]benzothieno[3, 2-b]benzothiophene (DPh-BTBT), ${ }^{27}$ and a polydiacetylene derivative (PDA), which was photopolymerized from 10,12pentacosadiynoic acid (PDA monomer) (Tokyo Chemical Industry Co.). Other than the PDA monomer, these organic materials were train-sublimated several times before use. The PDA monomer was used as purchased.

Using these six materials and metals with various work

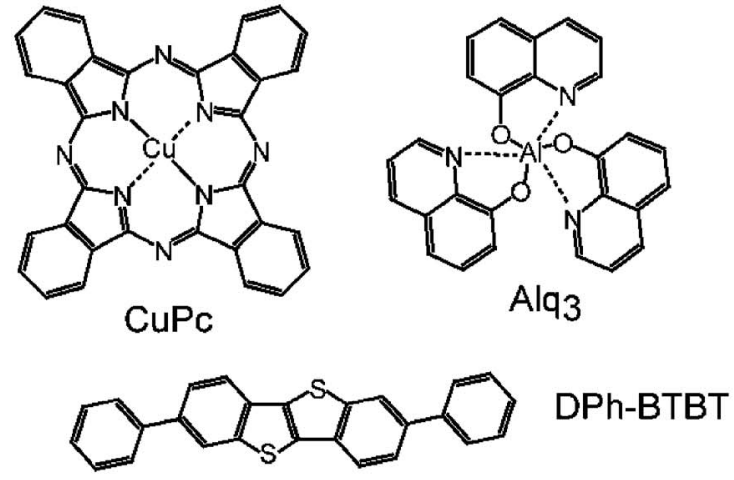

(a)

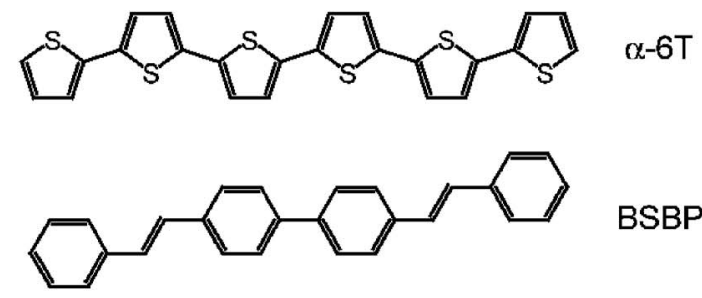

$\mathrm{H}_{25} \mathrm{C}_{12}=\mathrm{C}_{8} \mathrm{H}_{16} \mathrm{COOH}$ PDA monomer
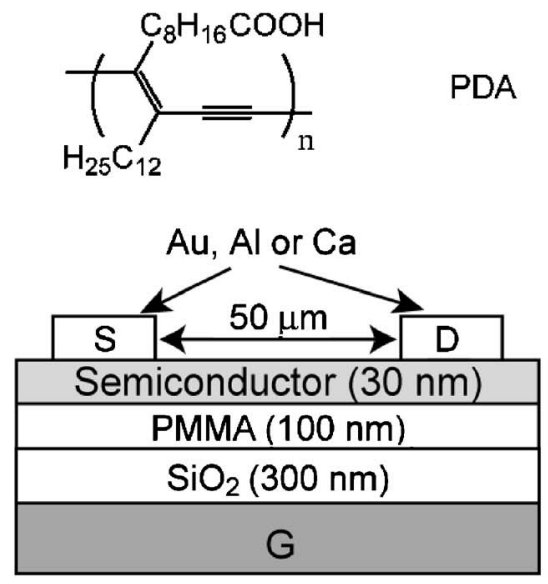

(b)

FIG. 1. (a) Molecular structures of organic semiconductors used. (b) Schematic view of OFET device structure.

functions for the contacts, we fabricated eight sample devices (the first eight listed in Table I). Figure 1(b) shows their schematic view. A 100 -nm-thick polymethylmethacrylate (PMMA) (Aldrich Co.) layer was dip coated onto $\mathrm{SiO}_{2} / p^{++}-\mathrm{Si}$ substrates to eliminate electron traps on the $\mathrm{SiO}_{2}$ surfaces.

We used a vacuum deposition and measurement system that consisted of one vacuum chamber each for organic deposition, metal deposition, and electrical measurement to control device preparation and measurement conditions. PMMA-coated substrates were first placed in the organic deposition chamber to form semiconducting layers. Organic semiconductor material was evaporated in a vacuum of 2 $\times 10^{-6} \mathrm{~Pa}$. The thickness and deposition rate were controlled at $30 \mathrm{~nm}$ and $0.05 \mathrm{~nm} / \mathrm{s}$, respectively. The substrates were held at room temperature during deposition. They were then 
TABLE I. Hole mobility, electron mobility, and threshold voltage for $n$ - and $p$-channel operations.

\begin{tabular}{lcccc}
\hline \hline \multicolumn{1}{c}{ Device } & $\begin{array}{c}\text { Hole mobility } \\
\left(\mathrm{cm}^{2} / \mathrm{V} \mathrm{s}\right)\end{array}$ & $\begin{array}{c}\text { Electron mobility } \\
\left(\mathrm{cm}^{2} / \mathrm{V} \mathrm{s}\right)\end{array}$ & $\begin{array}{c}\text { Threshold voltage } \\
(p \text {-channel }) \\
(\mathrm{V})\end{array}$ & $\begin{array}{c}\text { Threshold voltage } \\
(n \text {-channel }) \\
(\mathrm{V})\end{array}$ \\
\hline $\mathrm{PDA}(\mathrm{Au})$ & 0.12 & 0.025 & -60 & 31 \\
$\mathrm{CuPc}(\mathrm{Al})$ & $2.7 \times 10^{-5}$ & $7.5 \times 10^{-4}$ & -65 & 39 \\
$\mathrm{CuPc}(\mathrm{Au})$ & $1.7 \times 10^{-3}$ & $6.1 \times 10^{-5}$ & -30 & 57 \\
$\mathrm{BSBP}(\mathrm{Al})$ & $1.5 \times 10^{-3}$ & $2.5 \times 10^{-5}$ & -50 & 64 \\
$\mathrm{BSBP}(\mathrm{Au})$ & 0.030 & $9.0 \times 10^{-6}$ & -57 & 70 \\
$\mathrm{DPh}-\mathrm{BTBT}(\mathrm{Ca})$ & $4.4 \times 10^{-5}$ & $3.6 \times 10^{-4}$ & -79 & 77 \\
$\alpha-6 \mathrm{~T}(\mathrm{Ca})$ & $3.3 \times 10^{-4}$ & $2.5 \times 10^{-4}$ & -72 & 79 \\
$\mathrm{Alq}_{3}(\mathrm{Ca})$ & $\ldots$ & $5.5 \times 10^{-7}$ & $\cdots$ & 64 \\
$\mathrm{Alq}_{3}(\mathrm{Au} / \mathrm{Cr})^{\mathrm{b}}$ & $1.6 \times 10^{-7}$ & $\cdots$ & -43 & $\cdots$ \\
\hline
\end{tabular}

${ }^{\mathrm{a} A c t i v e ~ l a y e r ~ m a t e r i a l ~(s o u r c e ~ a n d ~ d r a i n ~ c o n t a c t ~ m e t a l) . ~}$

${ }^{\mathrm{b}}$ Bottom-contact electrodes on HMDS-treated substrate (channel width of $76 \mathrm{~mm}$ and channel length of $25 \mu \mathrm{m}$.)

transferred to the metal deposition chamber to prepare source and drain contacts. The channel length and width were $50 \mu \mathrm{m}$ and $6 \mathrm{~mm}$, respectively. The completed devices were then transferred to the measurement chamber to measure the FET characteristics. The measurement was done in a vacuum of $1 \times 10^{-5} \mathrm{~Pa}$. All processes after PMMA deposition were performed without breaking the vacuum.

We measured the electrical characteristics with a semiconductor parameter analyzer (Agilent 4156C). To control the vacuum pressure of the measurement chamber, we introduced air by tuning a leak valve and adjusting the rotation speed of a turbomolecular pump while monitoring the pressure.

\section{RESULTS AND DISCUSSION}

\section{A. FET characteristics measured without breaking vacuum}

Figures 2(a) and 2(b) show the transfer characteristics of the eight fabricated devices for $p$-channel and $n$-channel operation, respectively, measured without breaking the vacuum from device preparation through measurement. In both operation modes, current enhancement was observed in high gate-voltage ranges for most of the devices, indicating that the devices had ambipolar characteristics. Large current flows in low gate-voltage ranges originated from the opposite carrier flow, from the drain contact to the source contact, due to the high electric field between the drain and gate electrodes.

Among these devices, the PDA-based device with Au contacts showed the highest hole mobility of $0.12 \mathrm{~cm}^{2} \mathrm{~V}^{-1} \mathrm{~s}^{-1}$, and this value is of the same order as one previously reported. ${ }^{28}$ This PDA-based device also had a relatively high electron mobility of $0.025 \mathrm{~cm}^{2} \mathrm{~V}^{-1} \mathrm{~s}^{-1}$. These high mobilities are thought to be due to the high crystallinity of the film and the intrachain carrier transport along the polymer backbone. Furthermore, since the HOMO (5.6 $\mathrm{eV})$ and LUMO $(4.0 \mathrm{eV})$ levels $^{28}$ of the PDA are similar to the work function of $\mathrm{Au}(5.1 \mathrm{eV})$, electrons as well as holes can be easily injected from Au contacts. PDA-based OFETs with $\mathrm{UV} / \mathrm{O}_{3}$-treated $\mathrm{SiO}_{2} / p^{++} \mathrm{Si}$ substrates also showed similar ambipolar behavior although there were many hy- droxyl groups on the surface of the $\mathrm{SiO}_{2}{ }^{19}$ The long alkyl chain of the PDA would have kept the conjugated polymer backbone away from the hydroxyl groups.

Phthalocyanine derivatives show ambipolar behavior in FET structures. ${ }^{20,24,29}$ Yasuda et al. demonstrated ambipolar
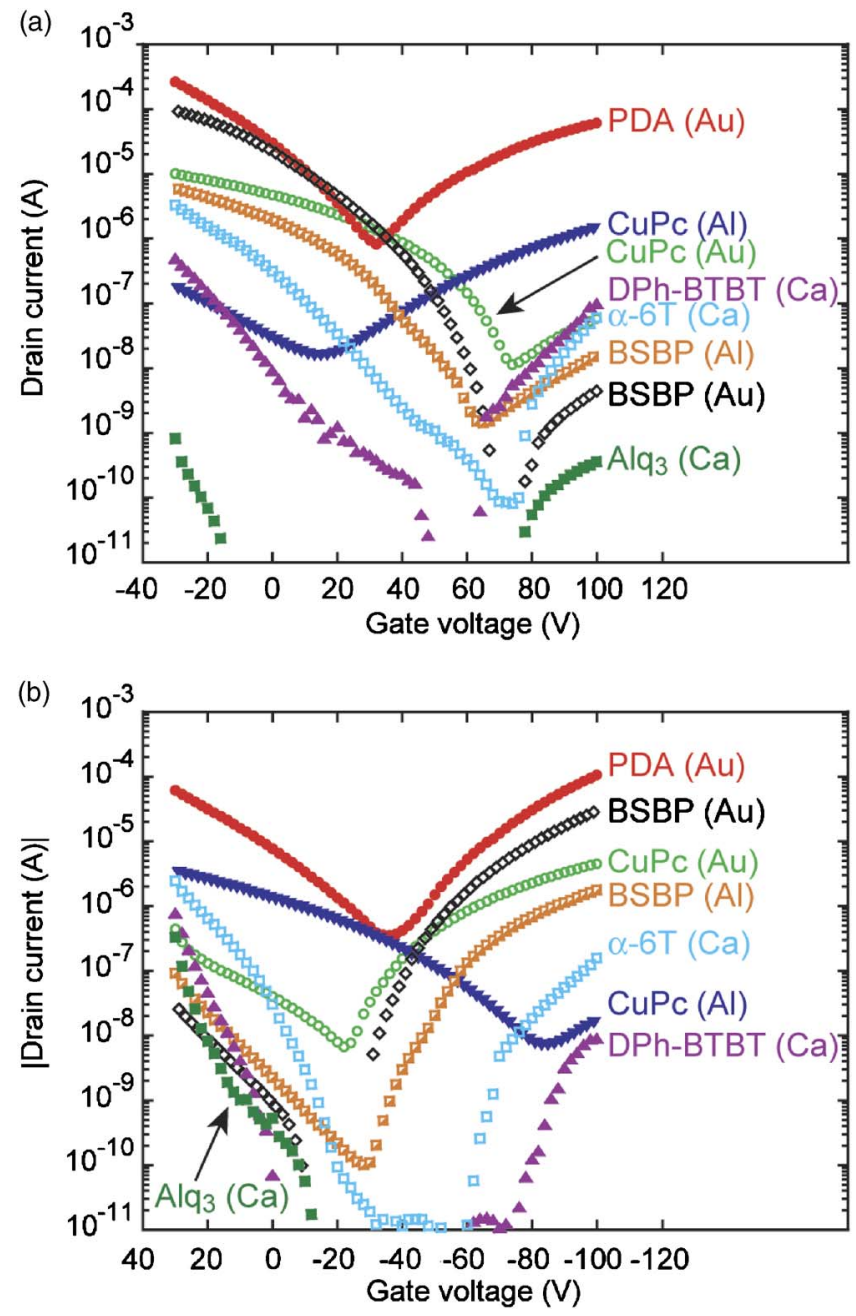

FIG. 2. (Color online) Transfer characteristics of eight devices for (a) $n$-channel and (b) $p$-channel operation. Drain voltage was set to -100 and $100 \mathrm{~V}$, respectively. Metals in parentheses indicate were used for source and drain contacts. 
carrier transport in CuPc-based OFETs with low work function $\mathrm{Ca}$ contacts, which reduced the electron injection barrier. ${ }^{29}$ In a similar manner, we also demonstrated ambipolar behavior when low work function $\mathrm{Al}$ contacts were used, as shown in Fig. 2(b). We even demonstrated ambipolar behavior when high work function Au contacts were used, although there is a large electron injection barrier of $1.4 \mathrm{eV}$ between the work function of $\mathrm{Au}(5.1 \mathrm{eV})$ and the LUMO level of $\mathrm{CuPc}(3.7 \mathrm{eV}) .{ }^{29}$ These results indicate that matching the work function of the metal contacts with the energy level of the organic semiconductor is not required for ambipolar operation if carriers can be injected from the electrodes. However, for $n$-channel operation, the drain current in the devices with Au contacts was smaller than that of the one with $\mathrm{Al}$ contacts, indicating that matching the work function is preferable for improving device performance. Thus, the apparent electron mobility of $6.1 \times 10^{-5} \mathrm{~cm}^{2} \mathrm{~V}^{-1} \mathrm{~s}^{-1}$ in the device with $\mathrm{Au}$ contacts was lower than that of 7.5 $\times 10^{-4} \mathrm{~cm}^{2} \mathrm{~V}^{-1} \mathrm{~s}^{-1}$ in the device with $\mathrm{Al}$ contacts.

Similar behaviors were also observed in the OFETs based on BSBP with a wide energy gap of $2.9 \mathrm{eV} .{ }^{14}$ Although the electron injection barrier from Au to the LUMO level of $\operatorname{BSBP}(2.1 \mathrm{eV})$ is very large (nearly $3 \mathrm{eV}$ ), we were able to observe $n$-type semiconducting behavior. Furthermore, we observed ambipolar behavior in the DPh-BTBT-based OFETs with Ca contacts, although DPh-BTBT has the widest energy gap $(3.1 \mathrm{eV})$ among the materials used in this study. These results clearly indicate that, in principle, ambipolar carrier transport can be achieved in almost all organic semiconductors.

In the $\alpha$-6T-based OFET with Ca contacts, we observed a high electron injection barrier although the work function of $\mathrm{Ca}(2.9 \mathrm{eV})$ is close to the LUMO level of the $\alpha-6 \mathrm{~T}$ [2.8 $\mathrm{eV}$ (Ref. 30)]. The threshold voltage for $n$-channel operation was as high as $79 \mathrm{~V}$, and the calculated electron mobility of $2.5 \times 10^{-4} \mathrm{~cm}^{2} \mathrm{~V}^{-1} \mathrm{~s}^{-1}$ was significantly lower than the reported hole mobility of $0.1-1.0 \mathrm{~cm}^{2} \mathrm{~V}^{-1} \mathrm{~s}^{-1}{ }^{31}$ There are two possible explanations for this phenomena. One is that there was unintentional doping in the bulk of the $\alpha-6 \mathrm{~T}$ layer and/or the $\alpha$-6T/PMMA interface was not eliminated even after several sublimations of the evaporation source. The other is that there was a positive vacuum level shift of $\alpha-6 \mathrm{~T}$ at the interface of $\mathrm{Ca} / \alpha-6 \mathrm{~T}$. Such positive vacuum level shifts of organic layers are thought to increase electron injection barriers. ${ }^{32}$

Although $\mathrm{Alq}_{3}$ is one of the most well known organic semiconductors, there have been no reports on preparing $\mathrm{Alq}_{3}$-based OFETs. By reducing the number of electron traps and injection barriers, we obtained $n$-channel FET characteristics in devices with Ca source and drain contacts. The fieldeffect mobility was $5.5 \times 10^{-7} \mathrm{~cm}^{2} \mathrm{~V}^{-1} \mathrm{~s}^{-1}$, which corresponds well with the value obtained by measuring the spacecharge-limited current in ultrahigh vacuum. ${ }^{33}$ However, it is significantly lower than the value obtained by time-offlight measurements, which was of the order of $10^{-5} \mathrm{~cm}^{2} \mathrm{~V}^{-1} \mathrm{~s}^{-1} \cdot{ }^{34,35}$ Nevertheless, the device showed no hole enhancement, as shown in Fig. 2(b). We had expected that $\mathrm{Alq}_{3}$ could transport holes as well as electrons in the devices we prepared. We therefore first changed the metal contacts from $\mathrm{Ca}$ to $\mathrm{Au}$ to reduce the hole injection barrier. However, it was difficult to obtain distinct $p$-channel FET characteristics due to the low drain current. We then prepared a bottom-contact-type OFET with interdigital electrodes of $\mathrm{Au} / \mathrm{Cr}$ (channel length of $76 \mathrm{~mm}$ and channel width of $25 \mu \mathrm{m})$ on a hexamethyldisylazane (HMDS) treated $\mathrm{SiO}_{2} / p^{++} \mathrm{Si}$ substrate to increase the drain current. With this sample, we observed apparent $p$-channel FET characteristics, indicating that $\mathrm{Alq}_{3}$ can intrinsically transport both holes and electrons. The FET behaviors of the $p$-channel $\mathrm{Alq}_{3}$-based OFET will be discussed in Sec. III B.

The field-effect mobilities of holes and electrons and the threshold voltages for the nine devices are summarized in Table I. Note that these mobilities are apparent values, simply calculated using a standard method in the saturation regime because these values usually contain contact resistances that originate from a mismatch between the work function of the metal contacts and the energy level of the organic semiconductor. As shown by the hole mobility of the CuPc-based OFET with Au contacts, the mobility in this study was relatively low compared to the value reported by Bao et al. $\left(0.02 \mathrm{~cm}^{2} / \mathrm{V} \mathrm{s}\right){ }^{36}$ This is because we did not optimize the deposition conditions. Optimization of the substrate temperature and deposition rate would improve the crystallinity and increase the grain size of the semiconducting films and lead to higher mobility.

\section{B. Effect of air exposure}

Here, we describe how carrier transport in the PDAbased ambipolar OFET and the $\mathrm{Alq}_{3}$-based $p$-channel OFET was affected by air. Figure 3 shows the vacuum pressure dependence of the transfer characteristics of the PDA-based ambipolar OFET in various environments from a high vacuum of $1 \times 10^{-5} \mathrm{~Pa}$ to atmospheric conditions (1 $\times 10^{5} \mathrm{~Pa}$ ). The vacuum pressure was controlled by introducing air into the measurement chamber. For $p$-channel operation, few changes were observed in the high vacuum range $\left(1 \times 10^{-5}-1 \times 10^{-1} \mathrm{~Pa}\right)$. When the vacuum pressure was lower than $1 \mathrm{~Pa}$, the drain current gradually increased as the vacuum decreased [Fig. 3(a), $p$-channel] due to the threshold shift from $-60 \mathrm{~V}$ for $1 \times 10^{-5} \mathrm{~Pa}$ to $-24 \mathrm{~V}$ for $1 \times 10^{5} \mathrm{~Pa}$ [Fig. 3(b), $p$-channel]. The change in hole mobility, from $0.12 \mathrm{~cm}^{2} \mathrm{~V}^{-1} \mathrm{~s}^{-1}$ for $1 \times 10^{-5} \mathrm{~Pa}$ to $0.096 \mathrm{~cm}^{2} \mathrm{~V}^{-1} \mathrm{~s}^{-1}$ for $1 \times 10^{5} \mathrm{~Pa}$, was small, as shown by the slopes of the square root drain currents for $p$-channel operation, which were almost the same. These results indicate that hole transport was not significantly affected by exposure to air. However, because oxygen molecules act as electron acceptors, ${ }^{26}$ the hole density in the PDA layer increased, causing a positive shift in the threshold voltage.

The drain current for $n$-channel operation dramatically decreased as vacuum pressure was reduced, indicating that oxygen or water molecules acted as traps. The mobility decreased (from 0.025 to $1.0 \times 10^{-6} \mathrm{~cm}^{2} \mathrm{~V}^{-1} \mathrm{~s}^{-1}$ ) as the pressure was reduced. However, the threshold voltage of around $35 \mathrm{~V}$ for $n$-channel operation was almost independent of the vacuum pressure, indicating that the electron density in the PDA layer was not affected by exposure to air. These find- 

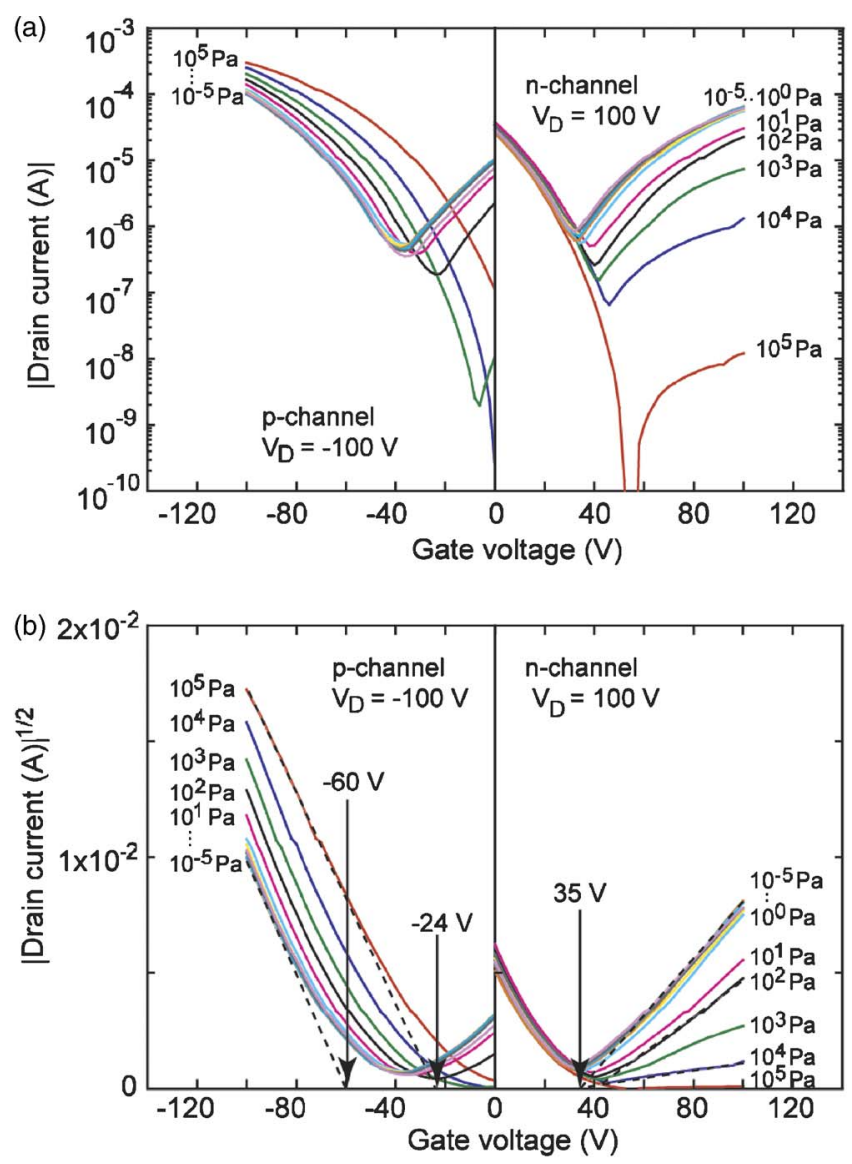

FIG. 3. (Color online) Vacuum-pressure dependence of transfer characteristics of PDA-based ambipolar OFET. (a) Drain current in logarithmic scale versus gate voltage. (b) Square-root drain current vs gate voltage. Drain voltage for $p$-channel and $n$-channel operation was set to -100 and $100 \mathrm{~V}$, respectively. Vacuum pressure was controlled from $1 \times 10^{-5}$ to $1 \times 10^{5} \mathrm{~Pa}$ (atmospheric pressure).

ings indicate that PDA film does not have an intrinsic electron charge and that accumulated electrons are injected from $\mathrm{Au}$ contacts. The introduction of donor gases such as $\mathrm{NH}_{3}$ may generate electron charges and should negatively shift threshold voltages. $^{23}$

Figure 4(a) shows the FET characteristics of the Alq $_{3}$-based bottom-contact-type FET for $p$-channel operation. The electrical measurements were carried out without breaking the vacuum after deposition. The device showed saturation behaviors with a field-effect hole mobility of 1.6 $\times 10^{-7} \mathrm{~cm}^{2} \mathrm{~V}^{-1} \mathrm{~s}^{-1}$, which is comparable to that of electrons. Here, the steep current increase at a gate voltage of zero was due to the electron flow from the drain contact to the source contact.

Cationic $\mathrm{Alq}_{3}$ has been thought to easily decompose due to the chemical reactions initiated by water and oxygen molecules. ${ }^{37,38}$ To clarify the effect of exposure to air on the $\mathrm{Alq}_{3}$-based $p$-channel OFET, we first exposed the device to air for $1 \mathrm{~h}$ and then returned it to a high vacuum. Almost no change was observed in the FET characteristics, indicating that no chemical reactions or contamination had occurred during the exposure. We then measured the device characteristics in air [Fig. 4(b)] and found that the current had significantly decreased and that the device less stably operated than
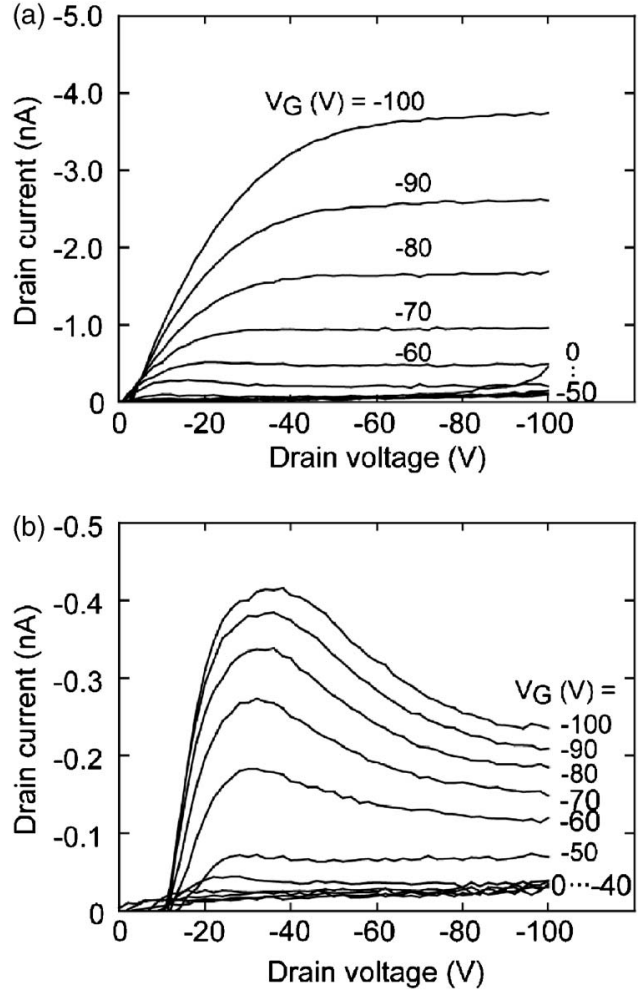

FIG. 4. FET characteristics of $\mathrm{Alq}_{3}$-based ambipolar OFET with HMDStreated $\mathrm{SiO}_{2} / p^{++} \mathrm{Si}$ substrate with bottom-contact electrodes of $\mathrm{Au} / \mathrm{Cr}$ (channel width of $76 \mathrm{~mm}$ and channel length of $25 \mu \mathrm{m}$ ) obtained in (a) vacuum of $1 \times 10^{-5} \mathrm{~Pa}$ and (b) air.

when it was in vacuum. The FET characteristics were not restored even when we returned the device to a high vacuum. These results indicate that measurement of the FET characteristics in air causes an irreversible chemical reaction between cationic $\mathrm{Alq}_{3}$ and oxygen and water molecules.

\section{SUMMARY}

To investigate the electrical characteristics of ambipolar OFETs, we used six organic semiconductor materials as the active layer: CuPc, $\alpha-6 \mathrm{~T}$, PDA, DPh-BTBT, BSBP, or $\mathrm{Alq}_{3}$. By controlling the device structure, device preparation, and measurement conditions, we found that these materials can transport both holes and electrons. Of these devices, the PDA-based FET showed the highest hole and electron mobilities ( 0.12 and $0.025 \mathrm{~cm}^{2} \mathrm{~V}^{-1} \mathrm{~s}^{-1}$, respectively). We found that the work function of the metal contacts and the energy gaps of the semiconductors are not essential for ambipolar operation. We also investigated the effect of exposure to air on PDA-based ambipolar OFETs. The hole mobility was barely affected by the exposure, while the electron mobility was significantly affected by it. However, the threshold voltage for $p$-channel operation was changed by exposure to air, while that for $n$-channel operation was independent of vacuum pressure. These behaviors indicate that the hole density in the active layer increased and that electron density was not affected by exposure to air. The FET characteristics of the $\mathrm{Alq}_{3}$-based $p$-channel OFET were not affected by exposure to air for $1 \mathrm{~h}$, while measurement in air degraded the FET characteristics. This indicates that measurement in air 
causes chemical reactions between cationic $\mathrm{Alq}_{3}$ and oxygen or water molecules. The preparation and measurement without breaking the vacuum enabled us to investigate both the intrinsic behavior and degradation processes and mechanisms of organic semiconductor materials.

\section{ACKNOWLEDGMENTS}

This work was supported by the Integrated Industry Academia Partnership (IIAP) of the Kyoto University International Innovation Center.

${ }^{1}$ C. D. Dimitrakopoulos and P. R. L. Malenfant, Adv. Mater. (Weinheim, Ger.) 14, 99 (2002).

${ }^{2}$ S. R. Forrest, Nature (London) 428, 911 (2004).

${ }^{3}$ H. Sirringhaus, T. Kawase, R. H. Friend, T. Shimoda, M. Inbasekaran, W. Wu, and E. P. Woo, Science 290, 2123 (2000).

${ }^{4}$ A. Dodabalapur, H. E. Katz, L. Torsi, and R. C. Haddon, Science 269, 1560 (1995).

${ }^{5}$ E. J. Meijer, D. M. de Leeuw, S. Setayesh, E. van Veenendaal, B.-H. Huisman, P. W. M. Blom, J. C. Hummelen, U. Scherf, and T. M. Klapwijk, Nat. Mater. 2, 678 (2003).

${ }^{6}$ C. Rost, D. J. Gundlach, S. Karg, and W. Rieß, J. Appl. Phys. 95, 5782 (2004).

${ }^{7}$ T. D. Anthopoulos, D. M. de Leeuw, E. Cantatore, S. Setayesh, E. J. Meijer, C. Tanase, J. C. Hummelen, and P. W. M. Blom, Appl. Phys. Lett. 85, 4205 (2004).

${ }^{8}$ Th. B. Singh, P. Senkarabacak, N. S. Sariciftci, A. Tanda, C. Lackner, R. Hagelauer, and G. Horowitz, Appl. Phys. Lett. 89, 033512 (2006).

${ }^{9}$ J. S. Swensen, C. Soci, and A. J. Heeger, Appl. Phys. Lett. 87, 253511 (2005).

${ }^{10}$ J. Zaumseil, R. H. Friend, and H. Sirringhaus, Nat. Mater. 5, 69 (2006).

${ }^{11}$ J. Zaumseil, C. L. Donley, J.-S. Kim, R. H. Friend, and H. Sirringhaus, Adv. Mater. (Weinheim, Ger.) 18, 2708 (2006).

${ }^{12}$ E. C. P. Smits, S. Setayesh, T. D. Anthopoulos, M. Buechel, W. Nijssen, R. Coehoorn, P. W. M. Blom, B. de Boer, and D. M. de Leeuw, Adv. Mater. (Weinheim, Ger.) 19, 734 (2007).

${ }^{13}$ K. Yamane, H. Yanagi, A. Sawamoto, and S. Hotta, Appl. Phys. Lett. 90, 162108 (2007).

${ }^{14}$ T. Sakanoue, M. Yahiro, C. Adachi, H. Uchiuzou, T. Takahashi, and A. Toshimitsu, Appl. Phys. Lett. 90, 171118 (2007).

${ }^{15}$ T. Takahashi, T. Takenobu, J. Takeya, and Y. Iwasa, Adv. Funct. Mater. 17, 1623 (2007).

${ }^{16}$ C. R. McNeill, A. Abrusci, J. Zaumseil, R. Wilson, M. J. McKiernan, J. H
Burroughes, J. J. M. Halls, N. C. Greenham, and R. H. Friend, Appl. Phys. Lett. 90, 193506 (2007).

${ }^{17}$ R. J. Chesterfield, C. R. Newman, T. M. Pappenfus, P. C. Ewbank, M. H. Haukaas, K. R. Mann, L. L. Miller, and C. D. Frisbie, Adv. Mater. (Weinheim, Ger.) 15, 1278 (2003).

${ }^{18}$ T. Yasuda, T. Goto, K. Fujita, and T. Tsutsui, Appl. Phys. Lett. 85, 2098 (2004).

${ }^{19}$ L.-L. Chua, J. Zaumseil, J.-F. Chang, E. C.-W. Ou, P. K.-H. Ho, H. Sirringhaus, and R. H. Friend, Nature (London) 434, 194 (2005).

${ }^{20}$ R. W. I. de Boer, A. F. Stassen, M. F. Craciun, C. L. Mulder, A. Molinari, S. Rogge, and A. F. Morpurgoa, Appl. Phys. Lett. 86, 262109 (2005).

${ }^{21}$ T. Takahashi, T. Takenobu, J. Takeya, and Y. Iwasa, Appl. Phys. Lett. 88, 033505 (2006).

${ }^{22}$ K. Kudo, T. Sumimoto, K. Hiraga, S. Kuniyoshi, and K. Tanaka, Jpn. J. Appl. Phys., Part 1 36, 6994 (1997).

${ }^{23}$ H. Tada, H. Touda, M. Takada, and K. Matsushige, J. Porphyr. Phthalocyanines 3, 667 (1999).

${ }^{24}$ H. Tada, H. Touda, M. Takada, and K. Matsushige, Appl. Phys. Lett. 76, 873 (2000).

${ }^{25}$ A. Tapponnier, I. Biaggio, and P. Gunter, Appl. Phys. Lett. 86, 112114 (2005).

${ }^{26}$ H. Graaf and D. Schlettwein, J. Appl. Phys. 100, 126104 (2006).

${ }^{27}$ K. Takimiya, H. Ebata, K. Sakamoto, T. Izawa, T. Otsubo, and Y. Kunugi, J. Am. Chem. Soc. 128, 12604 (2006).

${ }^{28}$ J. Nishide, T. Oyamada, S. Akiyama, H. Sasabe, and C. Adachi, Adv. Mater. (Weinheim, Ger.) 18, 3120 (2006).

${ }^{29}$ T. Yasuda, K. Fujita, and T. Tsutsui, Chem. Phys. Lett. 402, 395 (2005).

${ }^{30}$ The LUMO level of $\alpha-6 \mathrm{~T}$ was estimated by subtracting the energy gap obtained from the absorption edge from the HOMO level. The HOMO level of $\alpha-6 \mathrm{~T}(5.0 \mathrm{eV})$ was determined using ultraviolet photoemission spectroscopy (AC-1, Riken Keiki Co.).

${ }^{31}$ G. Horowitz, M. E. Hajlaoui, and R. Hajlaoui, J. Appl. Phys. 87, 4456 (2000).

${ }^{32}$ H. Ishii, K. Sugiyama, E. Ito, and K. Seki, Adv. Mater. (Weinheim, Ger.) 11, 605 (1999).

${ }^{33}$ M. Kiy, P. Losio, I. Biaggio, M. Koehler, A. Tapponnier, and P. Günter, Appl. Phys. Lett. 80, 1198 (2002).

${ }^{34}$ B. Chen and S. Liu, Synth. Met. 91, 169 (1997).

${ }^{35}$ S. Barth, P. Müller, H. Riel, P. F. Seidler, W. Rieß, H. Vestweber, and H. Bässler, J. Appl. Phys. 89, 3711 (2001).

${ }^{36}$ Z. Bao, A. J. Lovinger, and A. Dodabalapur, Appl. Phys. Lett. 69, 3066 (1996).

${ }^{37}$ F. Papadimitrakopoulos, X. M. Zhang, D. L. Thomsen, and K. A. Higginson, Chem. Mater. 8, 1363 (1996).

${ }^{38}$ H. Aziz, Z. D. Popovic, N. X. Hu, A. M. Hor, and G. Xu, Science 283, 1900 (1999). 